\title{
A SOFT CAPACITY METHOD FOR CDMA CELLULAR NETWORKS BASED ON ADAPTIVE HOMOGENOUS SIR THRESHOLD
}

\author{
S.Nourizadeh $^{1}$ and R.Tafazolli ${ }^{1}$ \\ ${ }^{1}$ CCSR, University of Surrey, Guildford GU2 7XH, England, S.Nourizadeh@eim.surrey.ac.uk
}

\begin{abstract}
It has been proven that the centralised method provides optimum control for radio resource management. In this paper, we propose a soft capacity method by means of a homogenous near-optimum SIR target algorithm for CDMA cellular systems based on distributed power control (DPC) methods. Simulation results prove that the system capacity (or resources) is maximised if the SIR target management at the load controller is considered. Moreover, during high cell load, by removing users experiencing the worse link loss, a gain in the capacity is observed as well as reducing the noise rise in the system compare to the classical DPC fixed SIR target.
\end{abstract}

Keywords - DPC, SIR target, System Level, Soft Capacity

\section{INTRODUCTION}

The capacity of a cellular CDMA-based system is interference limited, and requires power control to minimise the near-far effect problem, thereby maximising the capacity. [1] introduced an efficient (or optimal) method known as centralised power control applicable to FDMA and TDMA systems, where the concept of SIR balancing is applied and formulated as an eigenvalue problem. [2] introduced this optimum power control to terrestrial CDMA cellular systems. The major advantage of this technique is that it produces the maximum achievable SIR and the associated transmitter power of the mobiles. Nevertheless, its major drawback is the amount of information that needs to be communicated into the network and the significant computational effort required for each power control decision.

A distributed method achieving optimum SIR balancing is introduced in [3]. This approach provides an efficient way of solving the eigenvalue problem formulated in [1] without any centralised estimation procedure. The latter as well as the majority of the distributed methods in the literature, however, have concentrated their effort in the balancing problem and assumed a given SIR target. Traditionally, the SIR target considered in most of the proposed DPC is fixed and its value is provided by the link level requirement. Nevertheless a system capacity would be optimal, as stated in [1], if the SIR target was equal to the maximum achievable SIR of the system, which in counter part, requires centralized information. In literature, few have considered this users' SIR target variation at system level. [4] introduced the concept of graceful degradation of users SIR target according to their transmitter power levels. A more realistic approach of this principle (termed Soft Dropping) is discussed in [5]. Graceful degradation provides an effective way of reducing the probability that instability occurs. Transmitter power, however, does not comprise an optimum criterion for assessing whether the SIR target of a user should be increased or decreased. In an optimal scenario, the signal quality that users aim for (or SIR target) should be consistent with the network load and distribution of users in the system coverage area. An estimate of maximum achievable SIR in uplink and downlink (respectively $\gamma_{u}{ }^{*}$ and $\gamma_{d}^{*}$ in [1]) could be of great importance for assessing the network load and determining the upper limits of QoS (e.g. received SIR in CDMA) that the system is able to offer to all users. These estimates can then be exploited in the context of an outer loop function or used by a load control process, which may adjust the bit-rates of the users accordingly to the network congestion. Attempting to estimate $\gamma_{u}{ }^{*}$ and $\gamma_{d}{ }^{*}$ would be difficult, since estimating the path gain elements of the (time varying) link gain matrices would require a significant measurement effort. [8] demonstrated that a homogenous near optimal SIR target is possible by means of distributed method. In this paper, the near-optimum SIR target algorithm, presented in [8], is used to assess the system capacity by adapting the SIR target of the users, at system level, in function of the cell loads. This paper is presented as follow; first the homogenous nearoptimum SIR target algorithm introduced by [8] is presented and further extended with a stepwise removal algorithm. Finally the behaviour of the system capacity is simulated in a cellular environment for DPC with fixed SIR target and the near-optimum SIR target extended by stepwise removal.

\section{NEAR-OPTIMUM SIR TARGET POWER CONTROL}

\section{A. Algorithm Principle}

[1] and [2] explained that to obtain the maximum achievable SIR of the system, knowledge of the link gain matrix is necessary. Since the amount of information is difficult to acquire, [8] proposed a distributed method based on a single cell link losses information The objective of this algorithm is to allocate a SIR target to the BS as close as to the optimal SIR. Once the optimal SIR target set to all the BSs, by applying the DPC it is proven that the users transmit power will converge towards their optimum value [3]. 


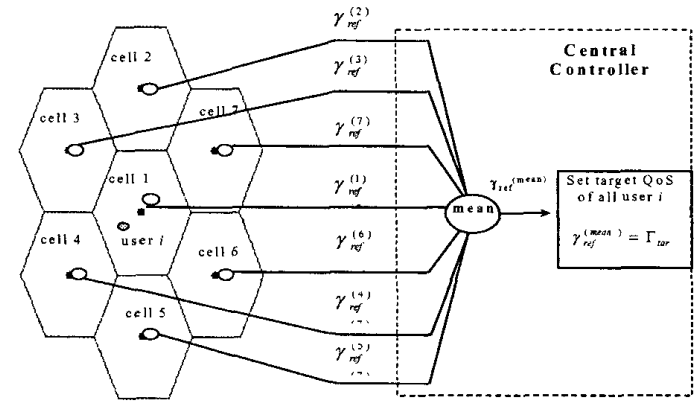

Figure 1: Near-Optimum SIR target DPC process

The algorithm in [8] performs the following steps:

1. BS (or cell site) measures the uplink-received powers and the average received SIR of intracell users. Each MS measures the average power of a pilot signal transmitted on the downlink of the active base station, compares that with the fixed pilot transmitter power provided by the downlink broadcast channel and determines the link gain (reciprocity is assumed between the uplink and the downlink). The link gains are reported to the base station and the transmitter powers of users are estimated.

2. The target SIR $\gamma_{R E F}$ is estimated separately by each BS and sent to the central controller (e.g. RNC in UMTS). This latter allocates an averaged value of the different estimated SIR target $\overline{\gamma_{R E F}}$ to the all the BS.

In a seven hexagonal cells topology as illustrated in Figure 1 , $\gamma_{R E F}^{(k)}$ (for $1 \leq k \leq 7$ ) is estimated separately at each BS, and the algorithm is invoked on the basis of the mean $\gamma_{R E F}$ value provided by a central controller to the cells.

\section{B. Algorithm Description}

The index of the user of interest is assumed to be $i$ in both the sets of $N$ users in the system and $N^{c_{i}}$ users in cell $c_{i}$. Let $G^{c_{i}}$ denote the uplink gain matrix of the one-cell system $c_{i}$, and $P^{c_{i}}$ denotes the uplink transmitter power vector of its users. If the measured intercell interference for the uplink at cell $c_{i}$ is denoted by $I_{\text {othercell }}^{c_{i}}$ and $n_{s}$ representing the background noise, then the average SIR of user $i$ can be expressed as:

$$
S I R_{i}=\frac{G_{i i}^{c_{i}} P_{i}^{c_{i}}}{\sum_{j \neq i}^{N^{c_{i}}} G_{i j}^{c_{i}} P_{j}^{c_{i}}+I_{\text {othercell }}^{c_{i}}+n_{s}}
$$

Based on the notation taken from [1] and [2] in one cell system $c_{i}, \lambda^{c^{*}}$ denotes the largest eigenvalue of $W^{c_{i}}$ and $v^{c_{i}^{*}}$ the corresponding eigenvector. $W^{c_{i}}$ represents the normalised uplink gain matrix for the cell $c_{i}$ and can be defined if the link gains between the base station in $c_{i}$ and all the mobile stations in this cell are known. Once the step 1 performed, the BS has knowledge of $W^{c_{l}}, v^{c_{i}{ }^{*}}$ (optimal transmit power, in one cell system $c_{i}$ ), and $P^{c_{i}}$ the actual transmit power of the MS in cell $c_{i}$. The objective is to estimate the near optimum transmit power $P^{c_{i}^{*}}$ as close as possible to $v^{*}$ (optimal transmit power) from the optimal intra cell transmit power $v^{c_{i}{ }^{*}} . P^{c_{i}^{*}}$ is in the form of:

$$
P^{c_{i}^{*}}=a^{c_{i}} v^{c_{i}^{*}}, a^{c_{i}}>0
$$

The value of $a^{c_{i}}$ provides a way of weighting the intracell optimum power vector $v^{c_{i}^{*}}$ against the currently measured $I_{\text {othercell }}^{c_{i}}$ and is specific to each BS. The selection of the parameter $a^{c_{i}}$ is of great significance. If $a^{c_{i}}$ is estimated perfectly, $P^{c_{i}^{*}}$ would converge towards $v^{*}$.

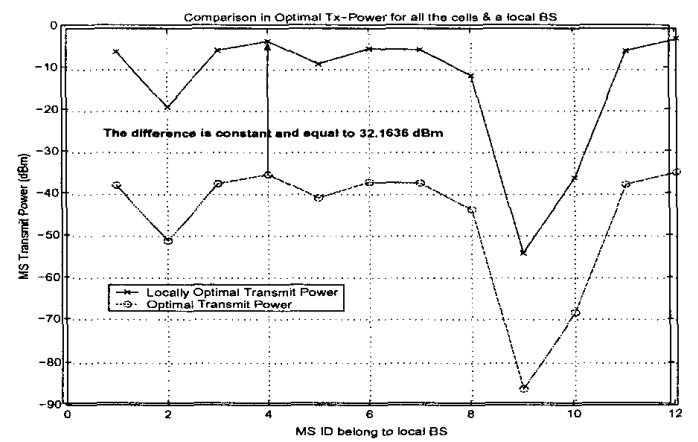

Figure 2: Optimal transmit power comparison in one cell and multi-cell system

In Figure 2, the values of $v^{*}$ (Optimal transmit power) corresponding to all cells and $v^{c_{i}^{*}}$ (Optimal transmit power for one cell system) are plotted for MS in the cell $c_{i}$. It can be seen that the value of $a^{c_{i}}$ is constant for all users that belong to the same cell. Nevertheless, we do not have 
knowledge of $v^{*}$ but only of $P^{c_{i}}$ the actual transmit power of the MS in cell $c_{i}$ in order to calculate the exact value of $a^{c_{i}}$. An efficient selection for $a^{c_{i}}$ is to choose the user with maximum actual transmitted power in cell $c_{i}$ over its estimated intracell optimum transmitted power:

$$
a^{c_{i}}=\frac{1}{\max \left(v^{c_{i}^{*}}\right)} \max \left(P^{c_{i}}\right)
$$

By including (3) into (2), $P^{c_{i}^{*}}$ is derived. Evidently, we can assume that factor $a^{c_{i}}$ is function of few parameters characterising the cellular system such as the number of MS per cell. The near-optimum SIR target $\gamma_{R E F}^{c_{i}}$ used to provide a criterion for the increase or decrease of $P^{c_{i}}$ according to (1), is re-written in function of $P^{c_{i}^{*}}$ as:

$$
\begin{aligned}
\gamma_{R E F}^{c_{i}} & =\frac{G_{i i}^{c_{i}} P_{i}^{c_{i}^{*}}}{\sum_{j \neq i}^{N^{c_{i}}} G_{i j}^{c_{j}} P_{j}^{c_{i}{ }^{*}}+I_{\text {othercell }}^{c_{i}}+n_{s}} \\
\gamma_{R E F}^{c_{i}} & =\frac{P_{i}^{c_{i}{ }^{*}}}{W^{{ }^{c_{i}}} P^{c_{i}{ }^{*}}+\frac{I_{\text {othercell }}^{c_{i}}+n_{s}}{G_{i i}^{c_{t}}}}
\end{aligned}
$$

(5) is obtained by including $W^{c_{i}}$ into (4). It should be noted that $\gamma_{R E F}^{c_{i}}$ is equal for all the intracell users since the optimum vector $P^{c_{i}{ }^{*}}$ provides received power balancing in the context of a one-cell system. Moreover, the balancing problem is particularly crucial for the intra cell users' transmit power. Nevertheless, the presence of the intercell interference forces all users to reduce their transmit power following (2).

\section{Stepwise Removal Algorithm}

If the cell experiences high cell loading, many MS will not be able to achieve the minimum SIR target $\gamma_{o}^{*}$ imposed by the link level. The interference produced by the transmit power of MS experiencing worse link loss is higher than the MSs which have lower link losses. By applying the proposed link loss estimation, the stepwise removal algorithm becomes an efficient method to remove the MS that cannot satisfy the minimum SIR target. We propose the following stepwise removal adapted to the near optimum algorithm:

\section{Algorithm:}

- Step 1: For each BS, determine the maximum achievable SIR level $\gamma_{U}^{*}$ from the near optimum algorithm. If $\gamma_{U}^{*} \geq \gamma_{o}^{*}$ use $\gamma_{U}^{*}$ as SIR target, else perform step 2 .

- Step 2: Remove mobile $k$ of a BS $c_{i}$ for which has the maximum of the row and column sums in the local normalised link gain matrix $W^{c_{i}}$. Perform step 1.

In this method, each BS will remove the MS that affects the cells' transmit power balancing. This is an efficient solution to optimise the system in a distributed way. It will assure that the received SIR of the non-removed MS will be equal at least to the minimum SIR target $\gamma_{o}^{*}$. This is not feasible with traditional DPC algorithm where no information on link gains is available.

\section{NUMERICAL RESULTS}

In this section we expose the numerical results of the dynamic simulator and of the flow chart diagram presented in Figure 3. The aim of our simulation is to observe the advantages of such SIR target estimation in the system capacity. The numerical results are given out for WCDMA systems assuming perfect SIR estimation and perfect intracell link loss estimation. The simulated system consists of 19 BS with cell radius of $2000 \mathrm{~m}$. The base station antenna height is $15 \mathrm{~m}$ above the average roof top level. The deployment scheme is assumed to have a hexagonal cell layout and omni-directional antennas are used.

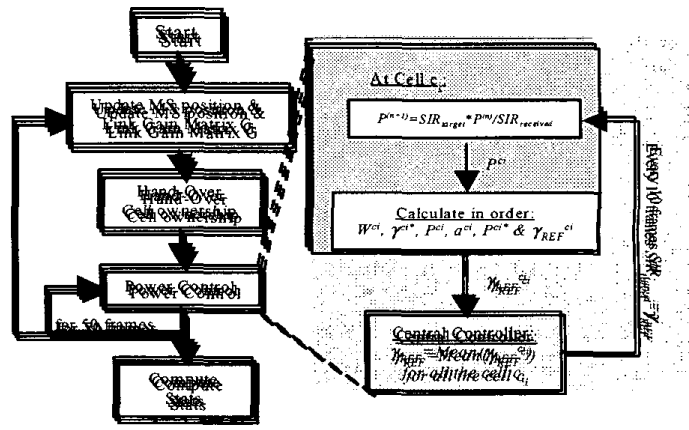

Figure 3: Flow chart diagram of Near-Optimum SIR target Algorithm

We consider that users are "immobile" during a snap shot (which lasts $500 \mathrm{~ms}$ until the next update of the MS positions) and therefore the link gain matrix of the system is assumed not to vary. Power control is performed in subsequent iterations and the transmitter power $P_{i}^{(n+1)}$ of each user $i$ is adjusted at iteration $n+1$ according to the classical DPC used in [3]: 


$$
P_{i}^{(n+1)}=\frac{\Gamma_{t \arg (i)}^{(n+1)}}{\gamma_{i}^{(n)}} P_{i}^{(n)}
$$

, where $\gamma_{i}^{(n)}$ is the measured SIR of the link at iteration $n$ and $\Gamma_{t \arg (i)}^{(n+1)}$ is the target SIR of user $i$ at iteration $n+1$. Each iteration lasts $10 \mathrm{~ms}$ which corresponds to the frame time in UMTS standard The outer loop function (or SIR target management at the load controller), which adjusts $\Gamma_{t \arg (i)}^{(n+1)}$ is invoked every 10 iterations and sets $\Gamma_{t \arg (i)}^{(n+1)}=\overline{\gamma_{R E F}}$ for all the users $i$ in the considered system. Note that in this paper, we assume that the intracell link gain estimation is perfect and each BS can measure the link loss of its MS from the transmitter power of the pilot channel.

The model defining the mobility of users in the Vehicular Test Environment is a pseudo-random mobility model with semi-directed trajectories. MSs' position is updated according to the decorrelation length used in the shadowing model and the direction that can be changed at each position update according to a probability of $20 \%$. The speed of mobile users is constant at $120 \mathrm{~km} / \mathrm{h}$ and the maximum angle of direction update is 45 degrees. All the assumptions and the models used in our dynamic system level simulator are based on [7] and summarised in Table 1.

Table 1: System Level Simulator Parameters

\begin{tabular}{|l|l|}
\hline \multicolumn{1}{|c|}{ Parameters } & \multicolumn{1}{c|}{ Settings } \\
\hline System scenario & UTRA FDD Uplink \\
\hline Environment & Suburban, Outdoor, Vehicular \\
\hline MS speed & $120 \mathrm{~km} / \mathrm{h}$ \\
\hline Simulation area & 19 omni-cells (2000m radius) \\
\hline Measure interval & $500 \mathrm{~ms}$ \\
\hline Traffic modelling & Speech $12.2 \mathrm{kbps}$ \\
\hline Bandwidth & $3.84 \mathrm{Mchips} / \mathrm{s}$ \\
\hline Handover Scheme & Hard Handover \\
\hline Handover hysterisis parameter & $5 \mathrm{~dB}$ \\
\hline Power control interval & $10 \mathrm{~ms}$ (or every frame) \\
\hline MS maximum transmit power & $21 \mathrm{dBm}$ \\
\hline Minimum SIR target & $-20.7 \mathrm{~dB}$ \\
\hline Mobility & UTRA model \\
\hline MS per Cell & 40 to $80 \mathrm{MS} /$ Cell \\
\hline Shadowing Standard deviation & $6 \mathrm{~dB}$ \\
\hline Shadowing Decorrelation length & $20 \mathrm{~m}$ \\
\hline Background noise & $-108 \mathrm{dBm}$ \\
\hline BS antenna gain & $13 \mathrm{dBi}$ \\
\hline
\end{tabular}

A single speech service of $12.2 \mathrm{kbps}$ was simulated and therefore a single homogenous SIR target was estimated for all MS in the 19 cells system. Simulations were run for 12,000 local mean SIR values. Each MSs' received SIR value is calculated after $500 \mathrm{~ms}$, which includes five updates of the homogenous SIR target, balancing of the MS transmitted power according to (6) and virtual hard handover. Heterogeneous SIR target for mixed services is a possible future work, however out of the scope of this paper.

The received SIR of all MSs were stored and the probability density function (PDF) of their average for different algorithms plotted. In Figure 4 the pdf of the received SIR of near-optimal and optimal scenarios without any constraints are plotted. The little difference between the curves is due to the SIR target error, proven in [8], to have a mean value in the order of $0.3 \mathrm{~dB}$ for any cell loads.

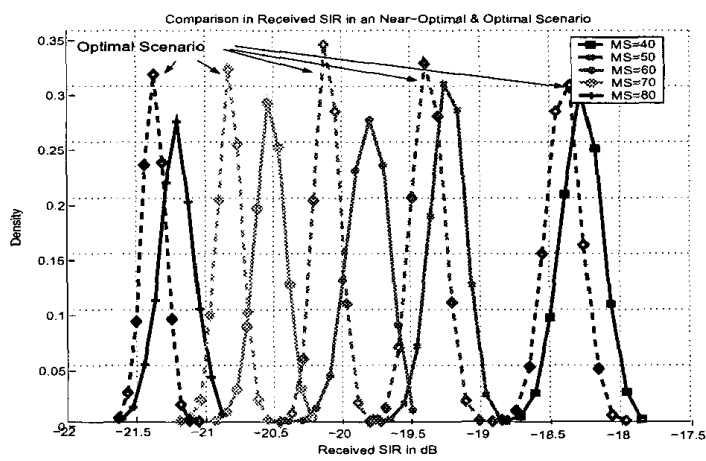

Figure 4: Comparison of the PDF of the average Received SIR Near-Optimum vs Optimal Power Control

From the Figure 4, it is observable that the optimal SIR target is function of the cell load. This provides an idea on soft capacity in CDMA is feasible by changing the threshold requirement and so by a load controller. Indeed, if there are fewer users in the system, a higher SIR target can be supported while if the number of MS increases, the SIR target of the system will have to be reduced.

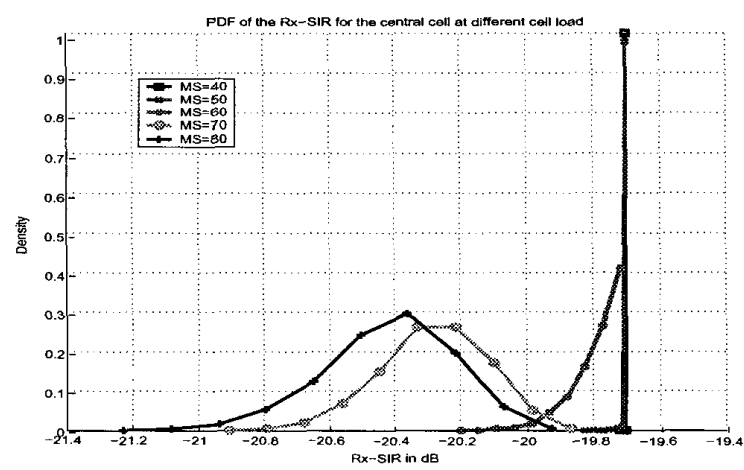

Figure 5: PDF of the Received SIR for Fixed Target DPC at different cell load 


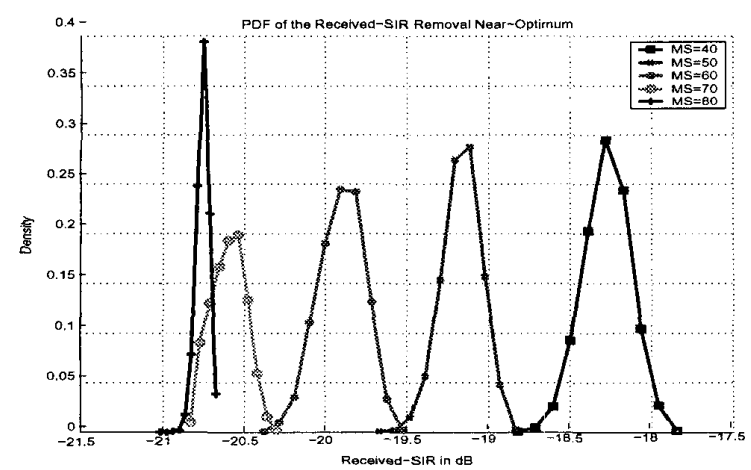

Figure 6: PDF of the Received SIR in Stepwise Removal Near Optimum Algorithm

In Figure 5 the received SIR of MS following the traditional DPC, where a fixed SIR target of $-19.7 \mathrm{~dB}$ is imposed for any cell load, are plotted. For low cell load (40 to $60 \mathrm{MS} / \mathrm{Cell}$ ) the received SIR achieve the fixed SIR target, however for higher cell load ( 70 to $80 \mathrm{MS} /$ Cell) in majority none of the MS can achieve the fixed target. Unlike the fixed target DPC algorithm, the stepwise removal algorithm has got a minimum SIR target, in our simulation chosen at $20.7 \mathrm{~dB}$, one $\mathrm{dB}$ margin less than the fixed target assumed in DPC. From Figure 6 it is seen that the adaptive SIR target algorithm provides flexibility and soft capacity to the system. Indeed, during low cell load, the system can support higher SIR and during high cell load, the system does not permit the SIR target to drop below a minimum value. It also maximise the system capacity by removing the MS that experiences the poorest link loss. The performance of the discussed algorithms are also compared in terms of outage probability, which is defined as the percentage of MS not satisfying the minimum SIR threshold (here assumed fixed at $-20.7 \mathrm{~dB}$ ). In Figure 7 , the outage probability of the stepwise removal near optimum threshold algorithm provides, as expected from the Figure 6, a gain of $15 \mathrm{MS}$ for an outage of $10 \%$ over the fixed SIR target (DPC method) for cell loads above $60 \mathrm{MS} /$ Cell.

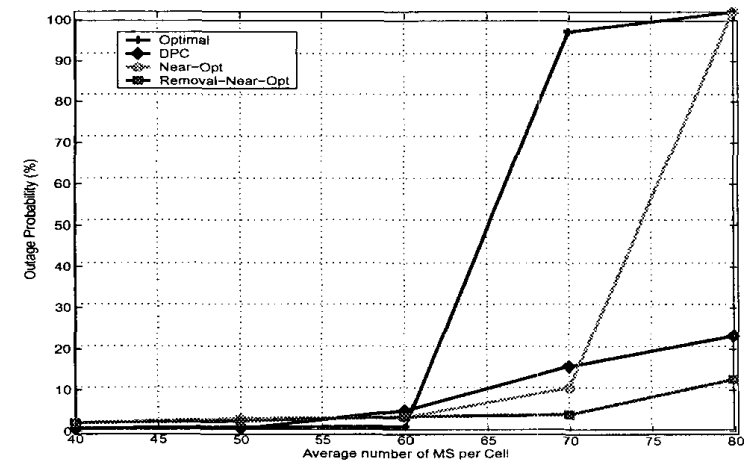

Figure 7: Outage Probability comparison of near-optimum \& optimum SIR target method
Another observation of the proposed scheme is presented in Figure 8 in terms of Noise Rise. The removal scheme allows MS to achieve high SIR if the cell is not fully loaded and this has for effect to increase the noise in the system, whereas during high cell loads, it minimises the noise by removing users experiencing worse link losses. An optimised algorithm on the SIR target in function of the noise rise is also feasible, however an upper SIR target value will have to be included in the algorithm.

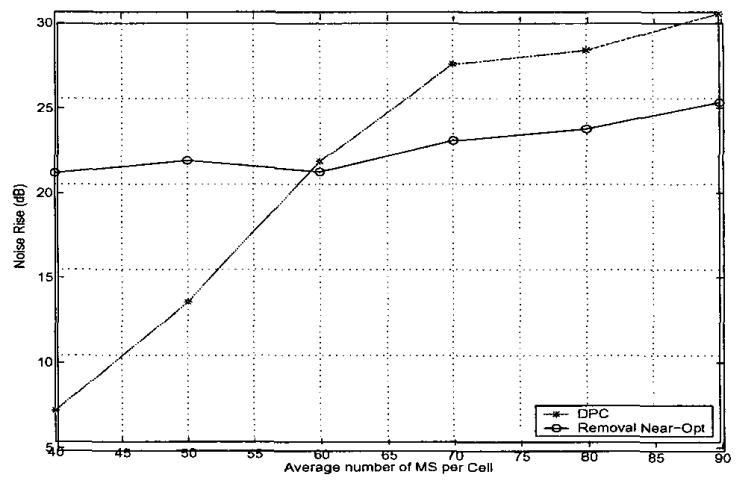

Figure 8: Noise Rise comparison

\section{CONCLUSION}

In this paper, a near-optimum SIR target power control algorithm that attempts to track the variation of the homogeneous threshold power control [2] was studied and compared to classical methods in literature. The proposed algorithm provides better knowledge of the system capacity and by applying an SIR target management at load controller, soft capacity is performed. Stepwise removal applied to the algorithm provides a gain in the capacity during high cell load. The implication of mixed services will force the system to consider heterogeneous SIR target.

\section{REFERENCES}

[1] J.Zander, "Performance of Optimum Transmitter Power Control in Cellular Radio Systems," IEEE VT, vol. 41, no. 1, pp.57-62, February 1992.

[2] Q.Wu, "Performance of Optimum Transmitter Power Control in CDMA Cellular Radio Systems," IEEE trans. VT, vol. 48, no. 2, pp.571-575, March 1999.

[3] J.Zander, "Distributed Cochannel Interference Control in Cellular Radio Systems," IEEE VT, vol. 41, no. 3, pp.305-311, August 1992.

[4] M.Almegren, H.Andersson and K. Wallstedt, "Power Control in a Celluar System," In Proc. IEEE VTC, Stockholm, Sweden, June 1994.

[5] R.D.Yates, S.Gupta and S. Sohn, "Soft Dropping Power Control," In Proc. IEEE VTC, Phoenix, USA, May 1997.

[6] J.Zander and M.Frodigh, "Comment on "Performance of Optimum Transmitter Power Control in Cellular Radio Systems'," IEEE VT, vol 43, no. 3, pp.636, August 1994.

[7] ETSI 101112 v3.2.0, Selection procedures for the choice of radio transmission technologies of the UMTS.

[8] S.Nourizadeh and R.Tafazolli, "Performance of an Near-Optimum SIR target Algorithm for CDMA cellular Networks" submitted to IEEE ISSSTA, August 2004, Sydney, Australia. 